\title{
The Method of Stationary Phase for Oscillatory Integrals on Hilbert Spaces
}

\author{
Jorge Rezende` \\ Fakultät für Physik, Universität Bielefeld, D-4800 Bielefeld 1, Federal Republic of Germany
}

\begin{abstract}
We develop the method of stationary phase for the normalizedoscillatory integral on Hilbert space, giving Borel summable expansions. The developments that we obtain hold for more general situations than the ones of previous papers on the same subject.
\end{abstract}

\section{Introduction}

In this paper we develop the method of stationary phase for oscillatory integrals on Hilbert spaces. Let us briefly describe the type of integrals we consider.

Let $\mathscr{H}$ be a real separable Hilbert space with inner product $(\cdot, \cdot) . \mathscr{M}(\mathscr{H})$ denotes the set of all bounded complex measures on $\mathscr{H}$ and $\mathscr{F}(\mathscr{H})$ denotes the set of their Fourier transforms: if $f \in \mathscr{F}(\mathscr{H})$, then $f: \mathscr{H} \rightarrow \mathbb{C}$ and

$$
f(x)=\int_{\mathscr{H}} \exp \{i(x, \alpha)\} d \mu(\alpha)
$$

for some $\mu \in \mathscr{M}(\mathscr{H})$.

In the following $B$ and $B^{-1}$ are bounded symmetric operators on $\mathscr{H}$. We study integrals of the form

$$
I(h)=\tilde{\int}_{\mathscr{H}} \exp \left\{\frac{i}{h} \phi(x)\right\} g(x) d x,
$$

where the integral is the normalized-oscillatory integral on $\mathscr{H}$ as defined in ref. [1], $\phi(x)$ is the real function $(1 / 2)(x, B x)-V(x)$ and $V, g \in \mathscr{F}(\mathscr{H})$.

Integrals of this form arise in the theory of Feynman path integrals; see e.g. refs. [1-7]. In this case the elements of $\mathscr{H}$ are the paths of finite kinetic energy, $\phi(x)$ is the action along the path $x, g$ is determined by the initial condition and $h$ is Planck's constant divided by $2 \pi$. The method of stationary phase for $(0.2)$ we shall develop, studying its asymptotic behaviour as $h \rightarrow 0$, is therefore connected with the heuristic ideas on the relation between quantum and classical mechanics.

* Supported by Deutscher Akademischer Austauschdienst (DAAD) 
To the method of stationary phase in finitely many dimensions, which is a classical object of study, new attention has been devoted in recent years in connection with the theory of pseudodifferential and Fourier integral operators. See e.g. refs. $[8,9,10]$.

Oscillatory integrals and the stationary phase method on Hilbert spaces have been studied in connection with application to Feynman path integrals in refs. $[2,3,4]$.

This is the first of a series of two papers. In the present paper we develop a general mathematical theory of stationary phase for integrals of the type $(0.2)$ giving Borel summable expansions in terms of powers of $h$, considering the case where $B>0$ and where $\phi$ has only one non-degenerate critical point. In particular the developments we obtain hold under more general situations than the ones in refs. $[2,3,4]$, which also did not discuss Borel summability. In the second paper we shall study the general situation where the phase function can have more than one stationary point.

Let us now briefly summarize the content of the different sections. In Sect. 1 we give the basic definitions of the oscillatory integrals. In Sect. 2 we give some technical results useful for the asymptotic expansions. In particular we establish several combinatorial formulae, we obtain bounds of some integrals, and we characterize a class of integrable functions. In Sect. 3 we study the case where the phase function $\phi$ has only one non-degenerate stationary point. We shall now briefly explain the main statements of this section.

Denote by $a$ the stationary point. In formula $(0.2)$ we can make the translation $x \rightarrow x+a$ without changing the value of $I(h)$, and we obtain

$$
I(h)=I^{*}(h) \exp \left\{\frac{i}{2 h}(a, B a)-\frac{i}{h} V(a)\right\},
$$

where

$$
I^{*}(h)=\int_{\mathscr{H}} \exp \left\{\frac{i}{2 h}(x, B x)-\frac{i}{h} W(x)\right\} g(x+a) d x
$$

and

$$
W(x)=V(x+a)-V(a)-(x, B a)
$$

Put now

$$
I^{*}(h)=\sum_{j=0}^{n-1} I_{j}^{*}(h)+\widetilde{R}_{n}(h)
$$

where

$$
I_{j}^{*}(h)=\frac{1}{j !}\left(-\frac{i}{h}\right)^{j} \int_{\mathscr{H}} \exp \left\{\frac{i}{2 h}(x, B x)\right\} W(x)^{j} g(x+a) d x .
$$

Under some assumptions on $V, g$ and $B$, one proves the following:

(a) $I(h)$ is defined and continuous in $\operatorname{Im} h \leqq 0, h \neq 0$, and is analytic in $\operatorname{Im} h<0$.

(b) Formula (0.3) is well defined in the region $\operatorname{Im} h<0$.

(c) $\bar{R}_{n}(h)$ converges uniformly to zero on compacts in the half plane $\operatorname{Im} h<0$. 
(d) $I_{j}^{*}(h)$ can be defined in $\operatorname{Im} h \leqq 0$, where it is $C^{\infty}$; this function is analytic in $\operatorname{Im} h<0$.

(e) $\sum_{j=0}^{\infty} I_{j}^{*}(h)$ converges absolutely and uniformly in $\operatorname{Im} h \leqq 0$.

From this we can infer that $\sum_{j=0}^{\infty} I_{j}^{*}(h)$ is a continuous function in $\operatorname{Im} h \leqq 0$, analytic in $\operatorname{Im} h<0$, which coincides with $I^{*}(h)$ in $\operatorname{Im} h \leqq 0, h \neq 0$. One can define $I^{*}(0)=\sum_{j=0}^{\infty} I_{j}^{*}(0)$.

The main theorem of this paper also establishes that $I^{*}(h)$ satisfies in $\operatorname{Im} h \leqq 0$ an estimate of the type

$$
\left|I^{*}(h)-\sum_{m=0}^{l-1} a_{m} h^{m}\right| \leqq A \sigma^{l}(l-1 / 2) !|h|^{l}
$$

(for $a_{m} \in \mathbb{C}, m \in \mathbb{N}, A, \sigma>0$ ) uniformly in $l \in \mathbb{N}$ and $h$. For the meaning of the coefficients $a_{m}$, see ref. [11].

From these properties we finally conclude that the formal power series $\Sigma a_{m} h^{m}$ is Borel summable.

\section{The normalized-Oscillatory Integral on Hilbert Space}

Let us begin recalling some definitions and properties stated in ref. [1]. One can define an algebra structure on $\mathscr{F}(\mathscr{H})$, respectively on $\mathscr{M}(\mathscr{H})$. We take as operations the sum of functions, respectively the sum of measures, the multiplication of a function, respectively a measure, by a complex number and the product of functions, respectively the convolution of measures.

If $f$ is the Fourier transform of $\mu$ we set

$$
\|f\|=\|\mu\|=\int_{\mathscr{H}} d|\mu|(\alpha)
$$

i.e. $\|\mu\|$, as well as $\|f\|$, is the total variation of the measure $\mu$. Under these definitions one can prove that $\mathscr{F}(\mathscr{H})$ and $\mathscr{M}(\mathscr{H})$ are Banach algebras, and it is clear that $(0.1)$ establishes an isomorphism between them.

Let $h \in \mathbb{R} \backslash\{0\}$ for general $B$ and $\operatorname{Im} h \leqq 0, h \neq 0$, if $B>0$. Let $f$ be the Fourier transform of $\mu \in \mathscr{M}(\mathscr{H})$. We define now the following normalized-oscillatory "integral" on $\mathscr{H}$ :

$$
\mathscr{F}(f)=\int_{\mathscr{H}} \exp \left\{\frac{i}{2 h}(x, B x)\right\} f(x) d x=\int_{\mathscr{H}} \exp \left\{-\frac{i h}{2}\left(\alpha, B^{-1} \alpha\right)\right\} d \mu(\alpha) .
$$

We remark that the integral with respect to $\mu$ is well defined as an integral on $\mathscr{H}$. The "integral" $\mathscr{F}(f)$ is a continuous bounded normalized linear functional on $\mathscr{F}(\mathscr{H})$ $(|\mathscr{F}(f)| \leqq\|f\|$ and $\mathscr{F}(1)=1)$. It follows from the fact that $\mathscr{F}(\mathscr{H})$ is a Banach algebra that sums and products of "integrable" functions are again "integrable" functions, and so are also compositions with entire functions. As a consequence one can easily see that $(0.2)$ is well defined. 
The formal notation involving $d x$ has the advantage of reminding us of the origins of the normalized-oscillatory integral and of its properties such as the invariance under translations and the Fubini theorem about iterated integration. For the proof and for a discussion of these and other properties of the normalizedoscillatory integral, see ref. [1]. See also refs. [2-5].

We shall also use some definitions and results stated in refs. [6,7], which we now briefly recall. Let $\varphi \in \mathscr{S}\left(\mathbb{R}^{m}\right), \varphi: \mathbb{R}^{m} \rightarrow \mathbb{C}$, and take $\varepsilon>0$ and $\rho \in \mathbb{R} \backslash\{0\}$. For a measurable map $f: \mathbb{R}^{m} \rightarrow \mathbb{C}$ we define

$$
l_{\varphi}^{\varepsilon}(f)=\int_{\mathbb{R}^{m}}(2 \pi i \rho)^{-m / 2} \exp \left\{\frac{i}{2 \rho}\langle x, x\rangle\right\} \varphi(\varepsilon x) f(x) d x,
$$

where $\langle\cdot, \cdot\rangle$ is the Euclidean inner product in $\mathbb{R}^{d}$, and $\zeta^{1 / 2}$ is chosen so that $\operatorname{Re} \zeta^{1 / 2} \geqq 0$ for $\operatorname{Re} \zeta \geqq 0$. If $l_{\varphi}(f)=\lim _{\varepsilon \downarrow 0} l_{\varphi}^{\varepsilon}(f)$ exists for all $\varphi \in \mathscr{P}\left(\mathbb{R}^{m}\right)$ with $\varphi(0)=1$ and has a value independent of $\varphi$, we say that $f \in \mathscr{I}^{\rho}\left(\mathbb{R}^{m} ; \mathbb{C}\right)$, and we write

$$
\mathscr{I}^{\rho}(f)=\int_{\mathbb{R}^{m}}^{0}(2 \pi i \rho)^{-m / 2}\left\{\frac{i}{2 \rho}\langle x, x\rangle\right\} f(x) d x=l_{\varphi}(f) .
$$

If $f$ has at most polynomial growth we can define for $\operatorname{Im} \zeta<0$,

$$
\mathscr{I}^{\zeta}(f)=(2 \pi i \zeta)^{-m / 2} \int_{\mathbb{R}^{m}} \exp \left\{\frac{i}{2 \zeta}\langle x, x\rangle\right\} f(x) d x .
$$

Let now $\mathscr{H}$ be the space of continuous functions $\gamma:[0, t] \rightarrow \mathbb{R}^{d}$, satisfying $\gamma(t)=0$, with $\gamma(\tau)=\left(\gamma^{1}(\tau), \ldots, \gamma^{d}(\tau)\right), \tau \in[0, t], \gamma^{i}$ absolutely continuous, $d \gamma^{i} / d \tau \in L^{2}[0, t]$, for $i=1, \ldots, d . \mathscr{H}$ is a real separable Hilbert space with inner product defined by

$$
\left(\gamma^{\prime}, \gamma\right)=\int_{0}^{t}\left\langle\frac{d \gamma^{\prime}}{d \tau}(\tau), \frac{d \gamma}{d \tau}(\tau)\right\rangle d \tau
$$

If $\pi=\left\{0=t_{0}<t_{1}<\cdots<t_{m(\pi)+1}=t\right\}$ is a finite partition of $[0, t]$ we define the piecewise linear approximation $P_{\pi}$ by

$$
\left(P_{\pi} \gamma\right)(s)=\gamma\left(t_{j}\right)+\left(s-t_{j}\right)\left[\gamma\left(t_{j+1}\right)-\gamma\left(t_{j}\right)\right]\left[t_{j+1}-t_{j}\right]^{-1}
$$

for $t_{j} \leqq s \leqq t_{j+1}, j=0,1, \ldots, m(\pi)$.

Consider now the complex Gaussian density $e_{h}: \mathscr{H} \rightarrow \mathbb{C}$ defined by

$$
e_{h}(\gamma)=\exp \left\{\frac{i}{2 h}(\gamma, \gamma)\right\}, \quad \operatorname{Im} h \leqq 0, h \neq 0 .
$$

For $f: \mathscr{H} \rightarrow \mathbb{C}$ we shall now define $\mathscr{F}^{h}(f)$. Put

$$
\mathscr{F}_{\pi}^{h}(f)=\int_{P_{\pi} \mathscr{H}}^{0}\left(f e_{h}\right) \uparrow_{P_{\pi} \mathscr{H}} d x \mid \int_{P_{\pi} \mathscr{H}}^{0} e_{h} \uparrow_{P_{\pi} \mathscr{H}} d x .
$$

Let $\delta(\pi)=\max \left\{t_{j+1}-t_{j} \mid j=0,1, \ldots, m(\pi)\right\}$. Then if $\lim _{\delta(\pi) \rightarrow 0} \mathscr{F}_{\pi}^{h}(f)$ exists, we say that $f$ is $\mathscr{F}^{h}$-integrable and write

$$
\mathscr{F}^{h}(f)=\lim _{\delta(\pi) \rightarrow 0} \mathscr{F}_{\pi}^{h}(f)
$$


One can prove $[6,7]$ that if $f \in \mathscr{F}(\mathscr{H})$ then $f$ is $\mathscr{F}^{h}$-integrable, and one obtains precisely

$$
\mathscr{F}^{h}(f)=\int_{\mathscr{H}} \exp \left\{\frac{i}{2 h}(x, x)\right\} f(x) d x .
$$

This is the reason why we shall use in this paper the right-hand side of (1.1) as a notation for $\mathscr{F}^{h}(f)$ even if $f \notin \mathscr{F}(\mathscr{H})$.

In the polygonal path formulation of the normalized-oscillatory integral one can prove also a translation formula. For more details see refs. $[6,7]$.

\section{Some Technical Results}

In this section we shall introduce notations and discuss some technical results, which will be needed to control the asymptotic expansion in the following section.

A) Some Combinatorial Analysis. As before $(\mathscr{H},(\cdot, \cdot))$ denotes a real separable Hilbert space. Let $x_{1}, \ldots, x_{2 n} \in \mathscr{H}$. We define $\left(x_{1}, \ldots, x_{2 n}\right)$ as the $2 n$-linear symmetric real function

$$
\left(x_{1}, \ldots, x_{2 n}\right)=\frac{1}{(2 n) !} \sum_{\sigma}\left(x_{\sigma(1)}, x_{\sigma(2)}\right) \cdots\left(x_{\sigma(2 n-1)}, x_{\sigma(2 n)}\right),
$$

where the summation is over all permutations $\sigma$ of $\{1, \ldots, 2 n\}$. The following convention will be useful:

$$
\left(x_{1}, \ldots, x_{k}, y_{1}, \ldots, y_{\lambda}\right)=\left(x^{k}, y_{1}, \ldots, y_{\lambda}\right)=\cdots=\left(y_{1}, \ldots, y_{\lambda}, x^{k}\right)
$$

whenever $x_{1}=\cdots=x_{k}=x$.

Consider now $\zeta \in \mathbb{C}$ and $f(\zeta)=\sum_{n=0}^{\infty} a_{n} \zeta^{n}$ an even, respectively odd, analytic function convergent for $|\zeta|<\rho$, with $\rho>0 ; f^{\prime}(\zeta)$ denotes the derivative of $f$, i.e. $f^{\prime}(\zeta)=\sum_{n=0}^{\infty} n a_{n} \zeta^{n-1}$; we also define $f^{(n+1)}(\zeta)=f^{(n) \prime}(\zeta)$. Let $k$ be an even (for $f$ even), respectively odd (for $f$ odd), integer and $x_{1}, \ldots, x_{k} \in \mathscr{H}$, For $x \in \mathscr{H},|x|=(x, x)^{1 / 2}<\rho$, we define the complex valued function

$$
f\left(x_{1}, \ldots, x_{k} ; x\right)=\sum_{n=0}^{\infty} a_{n}\left(x_{1}, \ldots, x_{k}, x^{n}\right) .
$$

It is now easy to see that

$$
D_{y} f\left(x_{1}, \ldots, x_{k} ; x\right)=f^{\prime}\left(x_{1}, \ldots, x_{k}, y ; x\right),
$$

where $D_{y}$ denotes the Fréchet derivative with respect to $x$ in the direction of the vector $y$.

We shall also need the following notations. Let $m \geqq 0$; for $m n \leqq s$, we define

$$
\begin{aligned}
& \left(x_{1}, \ldots, x_{k},\left(y_{1}+\cdots+y_{n}+z\right)^{s}\right)_{(y, m)} \\
& \quad=\sum_{\substack{\lambda_{1}+\\
\lambda_{1},+\lambda_{n}+\lambda=s}}\left(\lambda_{n}, \ldots, \lambda_{n}, \lambda\right)\left(x_{1}, \ldots, x_{k}, y_{1}^{\lambda_{1}}, \ldots, y_{n}^{\lambda_{n}} z^{\lambda}\right),
\end{aligned}
$$

and for $m n>s$ we assume that the right-hand side is equal to zero. 
For $m=0$ we have the multinomial theorem

$$
\left(x_{1}, \ldots, x_{k},\left(y_{1}+\cdots+y_{n}+z\right)^{s}\right)_{(y, 0)}=\left(x_{1}, \ldots, x_{k},\left(y_{1}+\cdots+y_{n}+z\right)^{s}\right) .
$$

One can now define

$$
f\left(x_{1}, \ldots, x_{k} ; y_{1}+\cdots+y_{n}+z\right)_{(y, m)}=\sum_{\lambda=0}^{\infty} a_{\lambda}\left(x_{1}, \ldots, x_{k},\left(y_{1}+\cdots+y_{n}+z\right)^{\lambda}\right)_{(y, m)} .
$$

For $m \geqq 1$ we have the following integral representation

$$
f\left(x_{1}, \ldots, x_{k} ; y+z\right)_{(y, m)}=\frac{1}{(m-1) !} \int_{0}^{1}(1-t)^{m-1} f^{(m)}\left(x_{1}, \ldots, x_{k}, y^{m} ; t y+z\right) d t .
$$

The proof of this formula is done considering first the case where $f(\zeta)=\zeta^{s}$, with $s \in \mathbb{N}$, using (2.1) and

$$
\int_{0}^{1} t^{k}(1-t)^{\lambda} d t=\frac{k ! \lambda !}{(k+\lambda+1) !}
$$

By induction over $n$ one can now prove

$$
\begin{aligned}
f( & \left.x_{1}, \ldots, x_{k} ; y_{1}+\cdots+y_{n}+z\right)_{(y, m)} \\
= & \frac{1}{(m-1) !^{n}} \int_{0}^{1} \cdots \int_{0}^{1}\left[\left(1-t_{1}\right) \ldots\left(1-t_{n}\right)\right]^{m-1} \\
& \quad \cdot f^{(m n)}\left(x_{1}, \ldots, x_{k}, y_{1}^{m}, \ldots, y_{n}^{m} ; t_{1} y_{1}+\cdots+t_{n} y_{n}+z\right) d t_{1} \ldots d t_{n} .
\end{aligned}
$$

Example 1.

$$
\begin{aligned}
\left(x_{1}+\cdots+x_{n}+y\right)_{(x, m)}^{s}= & \frac{s !}{(s-m n) !(m-1) !^{n}} \int_{0}^{1} \cdots \int_{0}^{1}\left[\left(1-t_{1}\right) \ldots\left(1-t_{n}\right)\right]^{m-1} \\
& \cdot\left(x_{1}^{m}, \ldots, x_{n}^{m},\left(t_{1} x_{1}+\cdots+t_{n} x_{n}+y\right)^{s-m n}\right) d t_{1} \ldots d t_{n} .
\end{aligned}
$$

Example 2. For $x \in \mathscr{H}$ consider the Gaussian $\exp \left\{-x^{2}\right\}$. We define

$$
D_{x_{1}} \ldots D_{x_{n}} \exp \left\{-x^{2}\right\}=(-1)^{n} H_{n}\left(x_{1}, \ldots, x_{n} \mid x\right) \exp \left\{-x^{2}\right\} \text {. }
$$

In this paper we shall use the convention $H_{n}\left(y_{1}, \ldots, y_{k}, x_{1}, \ldots, x_{\lambda} \mid x\right)=$ $H_{n}\left(y^{k}, x_{1}, \ldots, x_{\lambda} \mid x\right)=\cdots=H_{n}\left(x_{1}, \ldots, x_{\lambda}, y^{k} \mid x\right)$ whenever $y_{1}=\cdots=y_{k}=y$. If $\mathscr{H}$ is one-dimensional $H_{n}\left(x_{1}, \ldots, x_{n} \mid x\right)=x_{1} \ldots x_{n} H_{n}(x)$, where $H_{n}(x)$ is the usual Hermite polynomial of degree $n$, which is defined by

$$
H_{n}(x)=n ! \sum_{k=0}^{[n / 2]} \frac{(-1)^{k}(2 x)^{n-2 k}}{k !(n-2 k) !} .
$$

Note that in general $H_{n}\left(x_{1}, \ldots, x_{n} \mid x\right) \neq H_{n}\left(x_{1}, \ldots, x_{n} ; x\right)$. For $n=0,1,2,3$ we have:

$$
\begin{gathered}
H_{0}(x)=1, H_{1}\left(x_{1} \mid x\right)=2\left(x, x_{1}\right), H_{2}\left(x_{1}, x_{2} \mid x\right)=4\left(x, x_{1}\right)\left(x, x_{2}\right)-2\left(x_{1}, x_{2}\right), \\
H_{3}\left(x_{1}, x_{2}, x_{3} \mid x\right)=8\left(x, x_{1}\right)\left(x, x_{2}\right)\left(x, x_{3}\right)-12\left(x, x_{1}, x_{2}, x_{3}\right) .
\end{gathered}
$$

For $\zeta_{1}, \ldots, \zeta_{n} \in \mathbb{C}, x_{1}, \ldots, x_{n} \in \mathscr{H}$ we shall use the notation $\left(\zeta_{1} x_{1}, \ldots, \zeta_{n} x_{n}\right)=$ $\zeta_{1} \ldots \zeta_{n}\left(x_{1}, \ldots, x_{n}\right)$. With this convention and from formulae (2.2) and (2.4), one can 
easily establish the important relation

$$
\begin{aligned}
& \exp \left\{-\frac{i \zeta}{2}\left(x_{1}+\cdots+x_{n}+y\right)^{2}\right\}_{(x, m)} \\
& =\frac{1}{(m-1) !^{n} \int_{0}^{1} \cdots \int_{0}^{1}\left[\left(1-t_{1}\right) \ldots\left(1-t_{n}\right)\right]^{m-1}} \\
& \cdot H_{m n}\left(\left(\left(\frac{i \zeta}{2}\right)^{1 / 2} x_{1}\right)^{m}, \ldots,\left(\left(\frac{i \zeta}{2}\right)^{1 / 2} x_{n}\right)^{m} \mid\left(\frac{i \zeta}{2}\right)^{1 / 2}\left(t_{1} x_{1}+\cdots+t_{n} x_{n}+y\right)\right) \\
& \cdot \exp \left\{-\frac{i \zeta}{2}\left(t_{1} x_{1}+\cdots+t_{n} x_{n}+y\right)^{2}\right\} d t_{1} \ldots d t_{n}, \zeta \in \mathbb{C} .
\end{aligned}
$$

B) Bounds. We shall first compute some useful bounds for the "Hermite functions"

$$
F_{n}=\exp \left\{-\frac{i h}{2} y^{2}\right\} H_{n}\left(\left(\frac{i h}{2}\right)^{1 / 2} x_{1}, \ldots,\left(\frac{i h}{2}\right)^{1 / 2} x_{n} \mid\left(\frac{i h}{2}\right)^{1 / 2} y\right)
$$

with $x_{1}, \ldots, x_{n}, y \in \mathscr{H}$, as defined in the previous section and $\operatorname{Im} h \leqq 0$. Set $\operatorname{Im} h=$ $-|h| \delta$. From the definitions it follows immediately that

$$
\left|F_{n}\right| \leqq\left\{n !\left(\frac{|h|}{2}\right)^{n / 2}\left|x_{1}\right| \ldots\left|x_{n}\right| \sum_{\lambda=0}^{[n / 2]} \frac{\left[2(|h| / 2)^{1 / 2}|y|\right]^{n-2 \lambda}}{\lambda !(n-2 \lambda) !}\right\} \exp \left\{-\frac{|h| \delta}{2} y^{2}\right\} .
$$

Let $\eta \geqq 0$ and $k>0$; then $\eta^{k} \exp \{-\eta\} \leqq k^{k} \exp \{-k\}$. Let $\delta>0$ and put $\eta=$ $|h| \delta y^{2} / 2, k=n / 2-\lambda$. From (2.6) we get

$$
\left|F_{n}\right| \leqq\left|x_{1}\right| \ldots\left|x_{n}\right|\left(\frac{|h|}{2}\right)^{n / 2} n ! \sum_{\lambda=0}^{[n / 2]} \frac{[2(n-2 \lambda) / e \delta]^{n / 2-\lambda}}{\lambda !(n-2 \lambda) !}
$$

We shall now prove that

$$
n ! \sum_{\lambda=0}^{[n / 2]} \frac{[2(n-2 \lambda) / e \delta]^{n / 2-\lambda}}{\lambda !(n-2 \lambda) !} \leqq[4(1+\delta) / \delta]^{n / 2}(n / 2) !
$$

in order to obtain, from (2.7) and (2.8), the following bound:

$$
\left|F_{n}\right| \leqq\left|x_{1}\right| \ldots\left|x_{n}\right|[2|h|(1+\delta) / \delta]^{n / 2}(n / 2) !, \quad \operatorname{Im} h<0 .
$$

We recall first that

$$
(2 x) !=2^{2 x} x !(x-1 / 2) ! / \sqrt{\pi}, \quad \text { for } x \geqq 0,
$$

and (see ref. [12])

$$
x^{x} \sqrt{2 \pi x} \exp (-x) \leqq x ! \leqq x^{x} \sqrt{2 \pi x} \exp (-x+1 / 12 x), \text { for } x>0 .
$$

From (2.10) and (2.11) we easily get that

$$
2^{x}(x / 2)^{x / 2} \exp (-x / 2) / x ! \leqq \sqrt{\pi} /(x / 2-1 / 2) !, \quad x=0,1,2, \ldots
$$

and

$$
2^{x}(x / 2)^{x / 2} \exp (-x / 2) / x ! \leqq 1 /(x / 2) !, \quad x=0,1,2, \ldots
$$

For $n=2 p, p=0,1,2, \ldots$, and from (2.10) and (2.13), we get 


$$
\begin{aligned}
n ! \sum_{\lambda=0}^{[n / 2]} \frac{[2(n-2 \lambda) / e \delta]^{n / 2-\lambda}}{\lambda !(n-2 \lambda) !} & \leqq \frac{(2 p) !}{p !} \delta^{-p} \sum_{\lambda=0}^{p}\left(\begin{array}{l}
p \\
\lambda
\end{array}\right) \delta^{\lambda}=\frac{(p-1 / 2) !}{\sqrt{\pi}}\left(\frac{4(1+\delta)}{\delta}\right)^{n / 2} \\
& \leqq\left(\frac{n}{2}\right) !\left(\frac{4(1+\delta)}{\delta}\right)^{n / 2} .
\end{aligned}
$$

In the same way, for $n=2 p+1, p=0,1,2, \ldots$, and from (2.10) and (2.12) we obtain the inequality (2.8).

Finally we shall compute an upper bound for the integral

$$
I=\int \cdots \int\left|\alpha_{1}\right| \ldots\left|\alpha_{k}\right|\left(\left|\alpha_{1}\right| t_{1}+\cdots+\left|\alpha_{k}\right| t_{k}+|\beta|\right)^{m} \sum_{j=1}^{k} d|\mu|\left(\alpha_{j}\right) d t_{j} d|v|(\beta),
$$

where $\alpha_{1}, \ldots, \alpha_{k}, \beta \in \mathscr{H}, t_{1}, \ldots, t_{k} \in[0,1]$ and $\mu, v \in \mathscr{M}(\mathscr{H})$. Assume that

$$
\begin{array}{ll}
\int|\alpha|^{n} d|\mu|(\alpha) \leqq n ! L / \varepsilon^{n}, & 0<n \leqq m+1, \\
\int|\beta|^{n} d|v|(\beta) \leqq n ! M / \varepsilon^{n}, & 0 \leqq n \leqq m .
\end{array}
$$

First we remark the following; let $s, n \in \mathbb{N}$; the number of $n+1$-uples $\left(j_{0}, \ldots, j_{n}\right)$ such that $j_{0}+\cdots+j_{n}=s$, with $j_{0}, \ldots, j_{n} \in \mathbb{N}$, is $\left(\begin{array}{c}s+n \\ n\end{array}\right)$. The proof is done using the usual properties of the combinations. Now using formula (2.3) we get

$$
I=\frac{m !}{(m+k) !} \int \cdots \int\left(\left|\alpha_{1}\right|+\cdots+\left|\alpha_{k}\right|+|\beta|\right)_{(\alpha, 1)}^{m+k} \prod_{j=1}^{k} d|\mu|\left(\alpha_{j}\right) d|v|(\beta) \leqq \frac{(m+k) ! L^{k} M}{k ! \varepsilon^{m+k}}
$$

All these bounds will be needed later.

C) Integrable Functions. We give now a simple example of an integrable function [7]. Let $\alpha_{1}, \ldots, \alpha_{n}, \beta \in \mathscr{H}$. Then $\left(x, \alpha_{1}\right) \ldots\left(x, \alpha_{n}\right) \exp \{i(x, \beta)\}$ is integrable and

$$
\begin{aligned}
& \int_{\mathscr{H}} \exp \left\{\frac{i}{2 h} x^{2}\right\}\left(x, \alpha_{1}\right) \ldots\left(x, \alpha_{n}\right) \exp \{i(x, \beta)\} d x \\
& \quad=(i)^{n} \exp \left\{-\frac{i h}{2} \beta^{2}\right\} H_{n}\left(\left(\frac{i h}{2}\right)^{1 / 2} \alpha_{1}, \ldots,\left(\frac{i h}{2}\right)^{1 / 2} \alpha_{n} \mid\left(\frac{i h}{2}\right)^{1 / 2} \beta\right) .
\end{aligned}
$$

Obviously sums of functions of this type are also integrable. We have now the following lemma.

Lemma 1. Let $X$ be a metric space, $\omega$ a bounded complex measure defined on the Borel subsets of $X$ and $\operatorname{Im} h<0, h \neq 0$. We consider the measurable functions $g$, $f, f_{1}, \ldots, f_{p}: X \rightarrow \mathscr{H}$ such that $f, f_{1}, \ldots, f_{p}$ are bounded and

$$
\int_{X}|g(\xi)|^{j} d|\omega|(\xi)<\infty, \quad j=0,1, \ldots, p .
$$

This last condition can be dropped if $\operatorname{Im} h<0$. Then, the function $\varphi: \mathscr{H} \rightarrow \mathbb{C}$ defined by

$$
\varphi(x)=\int_{X}\left(x, f_{1}(\xi)\right) \ldots\left(x, f_{p}(\xi)\right) \exp \left\{i(x, g(\xi))+\frac{i}{h}(x, f(\xi))\right\} d \omega(\xi)
$$


is $\mathscr{F}^{h}$-integrable and

$$
\begin{aligned}
\int_{\mathscr{H}} \exp \left\{\frac{i}{2 h} x^{2}\right\} \varphi(x) d x=\int_{X} \exp \left\{-\frac{i}{2 h} f(\xi)^{2}-i(g(\xi), f(\xi))\right\} \\
\quad \cdot\left\{\int_{\mathscr{H}} \exp \left\{\frac{i}{2 h} x^{2}\right\}\left(x-f(\xi), f_{1}(\xi)\right) \ldots\left(x-f(\xi), f_{p}(\xi)\right) \exp \{i(x, g(\xi))\} d x\right\} d \omega(\xi),
\end{aligned}
$$

where the right-hand side is defined by (2.15). Sums of functions of this type are also integrable.

Proof. The lemma is proved in the same way, mutatis mutandis, as Proposition 5B in ref. [7]. The possibility of using Lebesgue's dominated convergence theorem is ensured by the conditions imposed on $g, f, f_{1}, \ldots, f_{p}$, taking into account the bounds (2.6) and (2.9). From these bounds we see also that the condition on $g$ is necessary if $\operatorname{Im} h=0$ and can be dropped if $\operatorname{Im} h<0$.

Remark. The class of functions $\varphi$ of the lemma is also closed under certain natural operations like translations and multiplications. Lemma 1 is necessary in order to justify formula (3.13) below.

\section{The Case of a Unique Non-Degenerate Stationary Point}

In this section we shall develop the method of the stationary phase for the integral $I(h)$ as defined by $(0.2)$ in the case where $B>0$ and the phase function $\phi(x)$ has a unique non-degenerate stationary point.

Consider the mapping $f(x) \rightarrow f_{B}(x)=f\left(B^{-1 / 2} x\right)$, which defines an automorphism of the Banach algebra $\mathscr{F}(\mathscr{H})$. One has immediately

$$
\int_{\mathscr{H}} \exp \left\{\frac{i}{2 h}(x, B x)\right\} f(x) d x=\mathscr{F}^{h}\left(f_{B}\right)=\int_{\mathscr{H}} \exp \left\{\frac{i}{2 h} x^{2}\right\} f_{B}(x) d x .
$$

As we see one can assume first that $B=1$ and later derive the results for general $B$ using the above defined automorphism.

From now on $V$ and $g$ are Fourier transforms of $\mu$ and $v$, respectively. Moreover we shall assume the existence of constants $L, M, \varepsilon>0$ such that

$$
\begin{array}{ll}
\int|\alpha|^{j} d|\mu|(\alpha) \leqq j ! L / \varepsilon^{j}, & 0<j, \\
\int|\beta|^{j} d|v|(\beta) \leqq j ! M / \varepsilon^{j}, & 0 \leqq j .
\end{array}
$$

Lemma 2. $V(x)$ is twice continuously Fréchet differentiable, $d^{2} V(x)$ is of trace class and

$$
\left\|d^{2} V(x)\right\|_{1} \leqq \int \alpha^{2} d|\mu|(\alpha),
$$

where $\|\cdot\|_{1}$ is the trace norm.

Proof. The proof follows as in ref. [2]. 
We remark that

$$
\begin{aligned}
(d V(x), y) & =i \int(\alpha, y) \exp \{i(x, \alpha)\} d \mu(\alpha), \\
\left(y, d^{2} V(x) z\right) & =-\int(\alpha, y)(\alpha, z) \exp \{i(x, \alpha)\} d \mu(\alpha),
\end{aligned}
$$

for every $y, z \in \mathscr{H}$.

Lemma 3. a) If there exists a $k>0$ such that $\left\|d^{2} V(x)\right\| \leqq k<1$ for every $x \in \mathscr{H}$, then the equation $d V(y)=y$ has a unique solution in $\mathscr{H}$. b) Let $V$ be such that $\int \alpha^{2} d|\mu|(\alpha)$ $<1$; then there exists a $k>0$ such that $\left\|d^{2} V(x)\right\| \leqq k<1$ for every $x \in \mathscr{H}$.

Proof. Part a) of the Lemma is contained in Lemma 2.3 in ref. [2]. For the proof of part b), take $k=\int \alpha^{2} d|\mu|(\alpha)$.

From now on we shall assume that $2 L<\varepsilon^{2}$, which implies that

$$
\int \alpha^{2} d|\mu|(\alpha)<1
$$

We see that (3.4) is a sufficient condition for the existence of a unique non-degenerate stationary point of the phase function, which we shall denote in the following by $a$. We shall also use the following notations: $V^{a}(x)=V(x+a), g^{a}(x)=g(x+a)$, $d \mu^{a}(\alpha)=\exp \{i(a, \alpha)\} d \mu(\alpha)$, and so on. From now on we define

$$
W(x)=V^{a}(x)-V(a)-(x, a) .
$$
write

Using the translation property of the normalized-oscillatory integral, we can

$$
I(h)=I^{*}(h) \exp \left\{\frac{i}{2 h} a^{2}-\frac{i}{h} V(a)\right\}
$$

where

$$
I^{*}(h)=\tilde{\int} \exp \left\{\frac{i}{2 h} x^{2}-\frac{i}{h} W(x)\right\} g^{a}(x) d x,
$$

with $W(x)$ defined by (3.5).

We are now going to investigate the asymptotic behaviour, as $h \rightarrow 0$, of the integral $I^{*}(h)$. Let us begin by introducing two functions: $\varphi_{n} \in \mathscr{F}^{h}(\mathscr{H})$, for $\operatorname{Im} h \leqq 0$, $h \neq 0$; and $\psi_{n} \in \mathscr{F}^{h}(\mathscr{H})$, for $\operatorname{Im} h<0$.

We recall first an integral representation which we shall often use

$$
\sum_{j \geqq n} \frac{\zeta^{j}}{j !}=\frac{\zeta^{n}}{(n-1) !} \int_{0}^{1}(1-t)^{n-1} \exp \{\zeta t\} \mathrm{dt}, \quad 0<n, \quad \zeta \in \mathbb{C} .
$$

Let

$$
\varphi_{n}(x)=W(x)^{n} g^{a}(x), \quad 0 \leqq n .
$$

From (3.5) and (3.6) we obtain the integral representation

$$
W(x)=-\int_{0}^{1} d t \int_{\mathscr{H}}(1-t)(x, \alpha)^{2} \exp \{i t(x, \alpha)\} d \mu^{a}(\alpha) .
$$


In order to apply Lemma 1, let

$$
\begin{aligned}
X & =(\mathscr{H} \times[0,1])^{n} \times \mathscr{H}, \quad \xi=\left(\alpha_{1}, t_{1}, \ldots, \alpha_{n}, t_{n}, \beta\right) \in X ; \\
f_{2 j-1}(\xi) & =f_{2 j}(\xi)=i \alpha_{j} /\left|\alpha_{j}\right|, \quad j=1, \ldots, n ; \\
f(\xi) & =0 ; \quad g(\xi)=t_{1} \alpha_{1}+\cdots+t_{n} \alpha_{n}+\beta ;
\end{aligned}
$$

and $\omega$ is the measure such that for $p: X \rightarrow \mathbb{C}$

$$
\int_{X} p(\xi) d \omega(\xi)=\int \cdots \int p(\xi) \prod_{j=1}^{n}\left|\alpha_{j}\right|^{2}\left(1-t_{j}\right) d \mu^{a}\left(\alpha_{j}\right) d t_{j} d v^{a}(\beta) .
$$

Let $\operatorname{Im} h \leqq 0, h \neq 0$. From Lemma 1 we get that $\varphi_{n} \in \mathscr{F}^{h}(\mathscr{H})$.

Now let

$$
\psi_{n}(x)=W(x)^{n} g^{a}(x) \int_{0}^{1}(1-t)^{n-1} \exp \left\{-\frac{i}{h} W(x) t\right\} d t, \quad 0<n .
$$

From (3.5) and (3.6) we obtain the integral representation

$$
W(x)=\int_{0}^{1} d t \int_{\mathscr{H}} i(x, \alpha) \exp \{i t(x, \alpha)\} d \mu^{a}(\alpha)-(x, a) .
$$

Define for $0 \leqq \lambda \leqq n$,

$$
\begin{aligned}
\psi_{n, \lambda}(x)= & {\left[\int_{0}^{1} d t \int(x, \alpha) \exp \{i t(x, \alpha)\} d \mu^{a}(\alpha)\right]^{\lambda}(x, a)^{n-\lambda} g^{a}(x) } \\
& \cdot \int_{0}^{1}(1-t)^{n-1} \exp \left\{-\frac{i}{h} W(x) t\right\} d t .
\end{aligned}
$$

Then

$$
\psi_{n}(x)=\sum_{\lambda=0}^{n} \frac{i^{\lambda}(-1)^{n-\lambda} n !}{\lambda !(n-\lambda) !} \psi_{n, \lambda}(x)
$$

Define now $\theta_{t} \in \mathscr{M}(\mathscr{H})$ such that

$$
\theta_{t}=\exp \left\{\frac{i}{h} \mathrm{~V}(a) t\right\} \sum_{l=0}^{\infty} \frac{1}{l !}\left(-\frac{i t}{h}\right)^{l}\left(\mu^{a}\right)^{* l} * v^{a}
$$

Then

$$
\left\|\theta_{t}\right\| \leqq\|v\| \exp \left\{-\frac{\delta}{|h|} V(a) t+\frac{\|\mu\|}{|h|} t\right\} \leqq\|v\| \exp \left\{\frac{\delta}{|h|}|V(a)|+\frac{\|\mu\|}{|h|}\right\},
$$

and $g^{a}(x)\left\{-(i / h)\left(V^{a}(x)-V(a)\right) t\right\}$ is the Fourier transform of $\theta_{t}$. Again, in order to apply Lemma 1 , let

$$
X=(\mathscr{H} \times[0,1])^{\lambda+1}, \quad \xi=\left(\alpha_{1}, t_{1}, \ldots, \alpha_{\lambda}, t_{\lambda}, \beta, t\right) \in X ;
$$

$f_{j}(\xi)=\alpha_{j} /\left|\alpha_{j}\right|, \quad j=1, \ldots, \lambda ; \quad f_{j}(\xi)=a, \quad j=\lambda+1, \ldots, n ; \quad f(\xi)=a t ; \quad g(\xi)=t_{1} \alpha_{1}+\cdots+$ $t_{\lambda} \alpha_{\lambda}+\beta$; and $\omega$ is the measure such that for $p: X \rightarrow \mathbb{C}$,

$$
\int_{X} p(\xi) d \omega(\xi)=\int(1-t)^{n-1} d t \int \cdots \int p(\xi) \prod_{j=1}^{\lambda}\left|\alpha_{j}\right| d \mu^{a}\left(\alpha_{j}\right) d t_{j} d \theta_{t}(\beta) .
$$


Let $\operatorname{Im} h<0$. From Lemma 1 we get that $\psi_{n, \lambda}, \psi_{n} \in \mathscr{F}^{h}(\mathscr{H})$. Let $n>0$. From formula (3.6) and Lemma 1 we get

$$
I^{*}(h)=\sum_{j=0}^{n-1} \frac{1}{j !}\left(-\frac{i}{h}\right)^{j} \tilde{\int} \exp \left\{\frac{i}{2 h} x^{2}\right\} \varphi_{j}(x) d x+\widetilde{R}_{n}(h),
$$

where

$$
\widetilde{R}_{n}(h)=\frac{1}{(n-1) !}\left(-\frac{i}{h}\right)^{n} \tilde{\int} \exp \left\{\frac{i}{2 h} x^{2}\right\} \psi_{n}(x) d x .
$$

As we have just proved (3.13) is well defined in the region $\operatorname{Im} h<0$.

Lemma 4. $\widetilde{R}_{n}(h)$ converges uniformly to zero on compacts in the half-plane $\operatorname{Im} h<0$.

Proof. We shall use the results of Sects. $2 B$ and $2 C$. We have

$$
\begin{aligned}
\tilde{R}_{n}(h)= & \left(-\frac{i}{h}\right)^{n} \int_{0}^{1} d t\left\{\int \frac{(1-t)^{n-1}}{(n-1) !} \exp \left\{-\frac{i t^{2}}{2 h} a^{2}\right\} \tilde{\int} \exp \left\{\frac{i}{2 h} x^{2}\right\}\right. \\
& \left.\cdot W(x-t a)^{n} \exp \{i(x, \beta)\} d x d \theta_{t}(\beta)\right\},
\end{aligned}
$$

where $\theta_{t}$ is defined by (3.11) with the substitutions $\mu^{a}$ by $\mu^{(1-t) a}$ and $v^{a}$ by $v^{(1-t) a}$. Then

$$
\begin{aligned}
& W(x-t a)^{n} \exp \{i(x, \beta)\} \\
& =\sum_{p+q+r+s=n} \frac{n !}{p ! q ! r ! s !}(-1)^{p+s} t^{q+s} i^{r+s}(x, a)^{p} a^{2 q} \\
& \quad \cdot \int \cdots \int\left(x, \alpha_{1}\right) \cdots\left(x, \alpha_{r}\right)\left(a, \gamma_{1}\right) \cdots\left(a, \gamma_{s}\right) \exp \left\{i \left(x, \alpha_{1} u_{1}+\cdots+\alpha_{r} u_{r}+\gamma_{1} v_{1}\right.\right. \\
& \left.\left.\quad+\cdots+\gamma_{s} v_{s}+\beta\right)\right\} \prod_{j=1}^{r} d \tilde{\mu}\left(\alpha_{j}, u_{j}\right) \prod_{j=1}^{s} d \tilde{\mu}\left(\gamma_{j}, v_{j}\right),
\end{aligned}
$$

where $\tilde{\mu}(\alpha, u)$ is a measure on $\mathscr{H} \times[0,1]$ such that for $p: \mathscr{H} \times[0,1] \rightarrow \mathbb{C}$,

$$
\int p(\alpha, u) d \tilde{\mu}(\alpha, u)=\iint p(\alpha, u) \exp \{-i t u(a, \alpha)\} d \mu^{a}(\alpha) d u \text {. }
$$

As before we put $\operatorname{Im} h=-\delta|h|$, with $\delta>0$. From (2.9), (3.12), (3.14) and (3.15), we get

$$
\begin{aligned}
\left|\tilde{R}_{n}(h)\right| \leqq|h|^{-n} \int_{0}^{1} d t\left\{\int \frac{(1-t)^{n-1}}{(n-1) !} \exp \left\{\frac{\delta}{2|h|} a^{2}\right\}\right. \\
\left.\cdot\left|\tilde{\int} \exp \left\{\frac{i}{2 h} x^{2}\right\} W(x-t a)^{n} \exp \{i(x, \beta)\} d x\right| d\left|\theta_{t}\right|(\beta)\right\} \\
\leqq|h|^{-n} \int_{0}^{1} d t \frac{(1-t)^{n-1}}{(n-1) !} \exp \left\{\frac{\delta}{2|h|} a^{2}\right\} \sum_{p+q+r+s=n} \frac{n !}{p ! q ! r ! s !} t^{q+s}|a|^{2 q+s+p} \\
\cdot\left(\frac{2|h|(1-\delta)}{\delta}\right)^{(p+r) / 2}\left(\frac{\mathrm{p}+r}{2}\right) ! \int \cdots \int \sum_{j=1}^{r+s}\left|\alpha_{j}\right| d|\mu|\left(\alpha_{j}\right) . \\
\cdot\|v\| \exp \left\{\frac{\delta}{|h|}|V(a)|+\frac{\|\mu\|}{|h|}\right\} .
\end{aligned}
$$

From $\left(\frac{p+r}{2}\right) !=\int_{0}^{+\infty} \exp \{-\eta\} \eta^{(p+r) / 2} d \eta$, we get 


$$
\left|\widetilde{R}_{n}(h)\right| \leqq \frac{K}{n !} \int_{0}^{+\infty} \exp \{-\eta\}\left(\eta^{1 / 2} P+Q+\eta^{1 / 2} R+S\right)^{n} d \eta,
$$

where

$$
\begin{aligned}
P & =|a|[2|h|(1+\delta) / \delta]^{1 / 2}, \\
Q & =a^{2}, \\
R & =[2|h|(1+\delta) / \delta]^{1 / 2} \int|\alpha| d|\mu|(\alpha), \\
S & =|a| \int|\alpha| d|\mu|(\alpha), \\
K & =|h|^{-n} \exp \left\{\frac{\delta}{2|h|} a^{2}+\frac{\delta}{|h|}|V(a)|+\frac{\|\mu\|}{|h|}\right\}\|v\| .
\end{aligned}
$$

From (3.16) we get immediately

$$
\begin{aligned}
\left|\tilde{R}_{n}(h)\right| & \leqq \frac{K}{n !} \int_{0}^{1} \exp \{-\eta\}(P+Q+R+S)^{n} d \eta \\
& +\frac{K}{n !} \int_{1}^{\infty} \exp \{-\eta\} \eta^{n / 2}(P+Q+R+S)^{n} \\
& \leqq \frac{K}{n !}\left(1-\frac{1}{e}+\left(\frac{n}{2}\right) !\right)(P+Q+R+S)^{n} .
\end{aligned}
$$

It is now easy to see that if $h$ belongs to a compact of the region $\operatorname{Im} h<0$, then $|h|$ and $\delta$ are bounded from below and above and the conclusion of the lemma is obvious.

It is natural now to investigate the asymptotic behaviour, as $h \rightarrow 0$, of the functions

$$
I_{n}^{*}(h)=\frac{1}{n !}\left(-\frac{i}{h}\right)^{n} \tilde{\int} \exp \left\{\frac{i}{2 h} x^{2}\right\} \varphi_{n}(x) d x, \quad \operatorname{Im} h \leqq 0, h \neq 0 .
$$

With the definition

$$
I_{n}^{*}(0)=\left(-\frac{1}{2}\right)^{n} \frac{1}{n !^{2}} \int \cdots \int\left(\sum_{j=1}^{n} \alpha_{j}+\beta\right)_{(\alpha, 2)}^{2 n} \prod_{j=1}^{n} d \mu^{a}\left(\alpha_{j}\right) d v^{a}(\beta),
$$

one has the following result.

Lemma 5. Under assumptions (3.1) and (3.2) $I_{n}^{*}(h), n \geqq 0$, is well defined in $\operatorname{Im} h \leqq 0$ analytic in $\operatorname{Im} h<0$ and $C^{\infty}$ on $\mathbb{R}$. We have in $\operatorname{Im} h \leqq 0$, for $l \geqq 0$,

$$
\begin{aligned}
I_{n}^{*}(h)= & \sum_{m=0}^{l-1} h^{m}\left(-\frac{i}{2}\right)^{m}\left(-\frac{1}{2}\right)^{n} \frac{1}{n !(m+n) !} \int \cdots \int\left(\sum_{j=1}^{n} \alpha_{j}+\beta\right)_{(\alpha, 2)}^{2 m+2 n} \\
& \cdot \prod_{j=1}^{n} d \mu^{a}\left(\alpha_{j}\right) d v^{a}(\beta)+R_{n, l}(h)
\end{aligned}
$$

and $R_{n, l}(h)$ satisfies the following estimate:

$$
\left|R_{n, l}(h)\right| \leqq \frac{M}{(2-\sqrt{2}) \sqrt{\pi}}\left(\frac{2 L(3+2 \sqrt{2})}{\varepsilon^{2}}\right)^{n}\left(\frac{2|h|}{\varepsilon^{2}(6-4 \sqrt{2})}\right)^{l} \frac{(n+l-1 / 2) !}{n !} .
$$


Moreover, for $2 L(3+2 \sqrt{2})<\varepsilon^{2}$, the series $\sum_{n=0}^{\infty} I_{n}^{*}(h)$ converges absolutely and uniformly in $\operatorname{Im} h \leqq 0$.

Proof. The fact that $I_{n}^{*}(h)$ is well defined in $\operatorname{Im} h \leqq 0, h \neq 0$, results from (3.1), (3.2) and Lemma 1 . We can now calculate $I_{n}^{*}(h)$ in two ways, using either the integral representation (3.10) or the integral representation (3.8) for $W(x)$. This gives, respectively

$I_{n}^{*}(h)=h^{-n} \sum_{k=0}^{n} \frac{i^{k}(-1)^{n-k}}{k !(n-k) !} \int \cdots \int \exp \left\{-\frac{i h}{2} A_{k}^{2}\right\} H_{n}^{(k)} \prod_{j=1}^{k} d \mu^{a}\left(\alpha_{j}\right) d t_{j} d v^{a}(\beta)$,
$I_{n}^{*}(h)=\frac{1}{n !}\left(-\frac{i}{h}\right)^{n} \int \cdots \int \exp \left\{-\frac{i h}{2} A_{n}^{2}\right\} H_{2 n}\left(1-t_{1}\right) \cdots\left(1-t_{n}\right) \prod_{j=1}^{n} d \mu^{a}\left(\alpha_{j}\right) d t_{j} d v^{a}(\beta)$,

where $A_{k}=\alpha_{1} t_{1}+\cdots+\alpha_{k} t_{k}+\beta, k=1, \ldots, n$,

$$
\begin{aligned}
& H_{n}^{(k)}=H_{n}\left(\left(\left(\frac{i h}{2}\right)^{1 / 2} a\right)^{n-k},\left(\frac{i h}{2}\right)^{1 / 2} \alpha_{1}, \ldots,\left(\frac{i h}{2}\right)^{1 / 2} \alpha_{k} \mid\left(\frac{i h}{2}\right)^{1 / 2} A_{k}\right), \\
& H_{2 n}=H_{2 n}\left(\left(\left(\frac{i h}{2}\right)^{1 / 2} \alpha_{1}\right)^{2}, \ldots,\left(\left(\frac{i h}{2}\right)^{1 / 2} \alpha_{n}\right)^{2} \mid\left(\frac{i h}{2}\right)^{1 / 2} A_{n}\right) .
\end{aligned}
$$

Comparing (2.5) and (3.18) we get

$$
I_{n}^{*}(h)=\frac{1}{n !}\left(-\frac{i}{h}\right)^{n} \int \cdots \int \exp \left\{-\frac{i h}{2}\left(\sum_{j=1}^{n} \alpha_{j}+\beta\right)^{2}\right\} \prod_{(\alpha, 2)}^{n} d \mu^{a}\left(\alpha_{j}\right) d v^{a}(\beta) .
$$

Now we have

$$
H_{n}^{(k)}=h^{n} \sum_{\lambda=0}^{[n / 2]} h^{-\lambda} a_{\lambda}^{(k)} \quad \text { and } \quad H_{2 n}=h^{n} \sum_{\lambda=0}^{n} h^{n-\lambda} a_{\lambda} .
$$

From (3.18), (3.19) and (3.20) it follows immediately that $I_{n}^{*}(h)$ is analytic in $\operatorname{Im} h<0$ and $C^{\infty}$ on $\mathbb{R}$. From (2.6) and $|a|=|d V(a)| \leqq L / \varepsilon$, we get

$$
\left|a_{\lambda}^{(k)}\right| \leqq \frac{n ! 2^{-\lambda}}{\lambda !(n-2 \lambda) !}\left(\frac{L}{\varepsilon}\right)^{n-k}\left|\alpha_{1}\right| \cdots\left|\alpha_{k}\right|\left(\left|\alpha_{1}\right| t_{1}+\cdots+\left|\alpha_{k}\right| t_{k}+|\beta|\right)^{n-2 \lambda} .
$$

From (3.6), (3.17) and (3.20), we get

$$
\left|I_{n}^{*}(h)-\sum_{m=-[n / 2]}^{l-1} b_{m} h^{m}\right| \leqq|h|^{l} \sum_{k=0}^{n} \sum_{\lambda=0}^{[n / 2]} \int \cdots \int \frac{\left(A_{k}^{2} / 2\right)^{\lambda+l}}{k !(n-k) !(\lambda+l) !}\left|a_{\lambda}^{(k)} \prod_{j=1}^{k} d\right| \mu\left|\left(\alpha_{j}\right) d t_{j} d\right| v \mid(\beta)
$$

for some $b_{m} \in \mathbb{C}, m=-[n / 2], \ldots, l-1$. From (3.6), (3.18) and (3.20), we get

$$
\begin{aligned}
\left|I_{n}^{*}(h)-\sum_{m=0}^{l-1} c_{m} h^{m}\right| \leqq & |h|^{l} \sum_{\lambda=0}^{n} \int \cdots \int \frac{\left(A_{n}^{2} / 2\right)^{-n+\lambda+l}}{n !(-n+\lambda+l) !} \\
& \cdot\left|a_{\lambda}\right|\left(1-t_{1}\right) \cdots\left(1-t_{n}\right) \prod_{j=1}^{n} d|\mu|\left(\alpha_{j}\right) d t_{j} d|v|(\beta)
\end{aligned}
$$

for some $c_{m} \in \mathbb{C}, m=0, \ldots, l-1$. Comparing now (3.22) and (3.23) we see that $b_{m}=0$ for $m=-[n / 2], \ldots,-1$ and $b_{m}=c_{m}$ for $m \in \mathbb{N}$. Comparing (3.19) and (3.23) we see 
that

$$
c_{m}=\left(-\frac{i}{2}\right)^{m}\left(-\frac{1}{2}\right)^{n} \frac{1}{n !(m+n) !} \int \cdots \int\left(\sum_{j=1}^{n} \alpha_{j}+\beta\right)_{(\alpha, 2)}^{2 m+2 n} \prod_{j=1}^{n} d \mu^{a}\left(\alpha_{j}\right) d v^{a}(\beta) .
$$

We need now to estimate the right-hand side of (3.22), i.e. $R_{n l}(h)$. From (2.14), (3.1), (3.2), (3.21) and (3.22), we get

$$
\left|R_{n, l}(h)\right| \leqq M\left(\frac{L}{2 \varepsilon^{2}}\right)^{n}\left(\frac{|h|}{2 \varepsilon^{2}}\right)^{l} n ! \sum_{k=0}^{n} \frac{(n+2 l+k) !}{k !^{2}(n-k) !} \sum_{\lambda=0}^{[n / 2]} \frac{2^{-2 \lambda+n}}{(\lambda+l) ! \lambda !(n-2 \lambda) !} .
$$

From

$$
\sum_{\lambda=0}^{[n / 2]} \frac{2^{-2 \lambda+n}}{(\lambda+l) ! \lambda !(n-2 \lambda) !}=\frac{(2 n+2 l) !}{n !(n+2 l) !(n+l) !}=\frac{2^{2 n+2 l}(n+l-1 / 2) !}{n !(n+2 l) ! \sqrt{\pi}}
$$

(see formula (3a) in ref. [13], p. 8) and from

$$
\sum_{k=0}^{n} \frac{(n+2 l+k) !}{k !^{2}(n-k) !} \leqq \frac{(n+2 l) !(3+2 \sqrt{2})^{n}}{n !(2-\sqrt{2})^{2 l+1}}
$$

the bound for $R_{n, l}(h)$ follows. The inequality (3.24) is proved in the following way. First we observe that

$$
\sum_{k=0}^{n} \frac{(n+2 l+k) !}{k !^{2}(n-k) !}=\frac{(n+2 l) !}{n !} \sum_{k=0}^{n}\left(\begin{array}{l}
n \\
k
\end{array}\right)\left(\begin{array}{c}
n+2 l+k \\
k
\end{array}\right) .
$$

It is clear now that $\sum_{k=0}^{n}\left(\begin{array}{l}n \\ k\end{array}\right)\left(\begin{array}{c}n+2 l+k \\ k\end{array}\right)$ is the coefficient of $z^{n}$ in the development in powers of $z$ of $(1+z)^{n}(1-z)^{-n-2 l-1},|z|<1$. Therefore

$$
\begin{aligned}
\sum_{k=0}^{n} \frac{(n+2 l+k) !}{k !^{2}(n-k) !} & =\frac{(n+2 l) !}{2 \pi i n !} \int_{|z|=r} \frac{(1+z)^{n}}{(1-z)^{n+2 l+1} z^{n+1}} d z \\
& \leqq \frac{(n+2 l) !(1+r)^{n}}{n !(1-r)^{n+2 l+1} r^{n}}, \quad \text { with } 0<r<1 .
\end{aligned}
$$

Choosing $r=\sqrt{2}-1$ to optimize the estimate, we obtain inequality (3.24). From the bound for $R_{n, l}(h)$ one gets

$$
\left|I_{n}^{*}(h)\right|=\left|R_{n, 0}(h)\right| \leqq \frac{M}{(2-\sqrt{2}) \sqrt{\pi}}\left(\frac{2 L(3+2 \sqrt{2})}{\varepsilon^{2}}\right)^{n} \frac{(n-1 / 2) !}{n !},
$$

and the final assertion of the lemma is easily established.

Lemma 6. Assume that $2 L<\varepsilon^{2}$. Then for every $m \geqq 0$ the series

$$
\left(-\frac{i}{2}\right)^{m} \sum_{n=0}^{\infty}\left(-\frac{1}{2}\right)^{n} \frac{1}{n !(m+n) !} \int \cdots \int\left(\sum_{j=1}^{n} \alpha_{j}+\beta\right)_{(\alpha, 2)}^{2 m+2 n} \prod_{j=1}^{n} d \mu^{a}\left(\alpha_{j}\right) d v^{a}(\beta)
$$

is absolutely convergent; moreover, for $m=0$, its value is given by

$$
\operatorname{Det}\left(1-d^{2} V(a)\right)^{-1 / 2} g(a) \text {, }
$$

where $\operatorname{Det}\left(\mathbb{1}-d^{2} V(a)\right)$ is the Fredholm determinant of the operator $\mathbb{1}-d^{2} V(a)$. 
Proof. By estimates of the type (2.14) we get

$$
\begin{aligned}
& \left|\int \ldots \int\left(\sum_{j=1}^{n} \alpha_{j}+\beta\right)_{(\alpha, 2)}^{2 m+2 n} \prod_{j=1}^{n} d \mu^{a}\left(\alpha_{j}\right) d v^{a}(\beta)\right| \\
& \leqq(2 m+2 n) ! \sum_{\substack{j_{1}+j_{n}+j=2 m+2 n \\
j_{1}, \cdot j_{n} \geqq 2}} \int \cdots \int \frac{\left|\alpha_{1}\right|^{j_{1}} \cdots\left|\alpha_{n}\right|^{j n}|\beta|^{j}}{j_{1} ! \cdots j_{n} ! j !} \\
& \cdot \prod_{k=1}^{n} d|\mu|\left(\alpha_{k}\right) d|v|(\beta) \leqq \frac{(2 m+2 n) !(2 m+n) ! L^{n} M}{n !(2 m) ! \varepsilon^{2 m+2 n}} .
\end{aligned}
$$

Now it is trivial to check the absolute convergence of the series if $2 L<\varepsilon^{2}$.

In order to compute the value of the series for $m=0$ let us first remark that $\left(\sum_{j=1}^{n} \alpha_{j}+\beta\right)_{(\alpha, 2)}^{2 n}=\left(\sum_{j=1} \alpha_{j}\right)_{(\alpha, 2)}^{2 n}$. In this case the series is reduced to

$$
\sum_{n=0}^{\infty}\left(-\frac{1}{2}\right)^{n} \frac{1}{n !^{2}} \int \cdots \int\left(\sum_{j=1}^{n} \alpha_{j}\right)_{(\alpha, 2)}^{2 n} \prod_{j=1}^{n} d \mu^{a}\left(\alpha_{j}\right) g(a) .
$$

Put

$$
I_{n}=\int \cdots \int\left(\sum_{j=1}^{n} \alpha_{j}\right)_{(\alpha, 2)}^{2 n} \prod_{j=1}^{n} d \mu^{a}\left(\alpha_{j}\right)
$$

and

$$
A_{n}=\int \cdots \int\left(\alpha_{1}, \alpha_{2}\right) \cdots\left(\alpha_{n-1}, \alpha_{n}\right)\left(\alpha_{n}, \alpha_{1}\right) \prod_{j=1}^{n} d \mu^{a}\left(\alpha_{j}\right)
$$

We have then that

$$
I_{n}=\sum_{\substack{l_{1}<<<l_{k} \\ l_{1} j_{1}+}+l_{k} k_{k}=n} A_{i_{1}}^{j_{1}} \cdots A_{i_{k}}^{j_{k}} \frac{n !^{2} 2^{n-\left(j_{1}+j_{k}\right)}}{i_{1}^{j_{1}} \cdots i_{k}^{j_{k}} j_{1} ! \cdots j_{k} !}
$$

Hence

$$
\sum_{n=0}^{\infty} \frac{I_{n}}{(-2)^{n} n !^{2}}=\sum_{n=0}^{\infty}(-1)^{n} \sum_{\substack{i_{1}<<\\ i_{1} j_{1}+}+i_{k} j_{k}=n} \frac{2^{-\left(j_{1}++j_{k}\right)}}{i_{1}^{j_{1}} \cdots i_{k}^{j_{k}} j_{1} ! \cdots j_{k} !} A_{i_{1}}^{j_{1}} \cdots / j_{i_{k}}^{j_{k}} .
$$

Now put $T=d^{2} V(a)$. Then from (3.3) we get

$$
(x, T y)=-\int(x, \alpha)(y, \alpha) d \mu^{a}(\alpha)
$$

and also

$$
\left(x, T^{n} y\right)=(-1)^{n} \int \cdots \int\left(x, \alpha_{1}\right) \cdots\left(\alpha_{n-1}, \alpha_{n}\right)\left(\alpha_{n}, y\right) \prod_{j=1}^{n} d \mu^{a}\left(\alpha_{j}\right)
$$

This implies

$$
\operatorname{tr} T^{n}=(-1)^{n} \sum_{i=1}^{\infty} \int \cdots \int\left(e_{i}, \alpha_{1}\right) \cdots\left(\alpha_{n-1}, \alpha_{n}\right)\left(\alpha_{n}, e_{i}\right) \prod_{j=1}^{n} d \mu^{a}\left(\alpha_{j}\right)=(-1)^{n} A_{n}
$$

where $\left(e_{i}\right)_{i=1, . .}$ is any orthonormal basis of $\mathscr{H}$. 
From the assumption of the lemma and from Lemma 2 we get that $\|T\|<1$. Hence $\operatorname{Det}(\mathbb{1}-T)^{-1 / 2}$ exists and we have

$$
\operatorname{Det}(1-T)^{-1 / 2}=\exp \{-(1 / 2) \operatorname{tr} \log (1-T)\}
$$

From (3.26) and (3.27) we get

$$
\operatorname{Det}(1-T)^{-1 / 2}=\exp \left\{\frac{1}{2} \sum_{n=1}^{\infty} B_{n}\right\}=\sum_{i=0}^{\infty}\left(\frac{1}{2}\right)^{i} \sum_{j_{1}+} \frac{B_{1}^{j_{1}} \cdots B_{n}+=i}{j_{n} ! \cdots j_{n} ! \cdots},
$$

where only a finite number of $j_{n}$ are different from zero and $B_{n}=(-1)^{n} A_{n} / n$. Then

$$
\begin{aligned}
\operatorname{Det}(1-T)^{-1 / 2} & =\sum_{n=0}^{\infty} \sum_{\substack{i_{1}<<<i_{k} \\
i_{1} j_{1}+}+i_{k} k_{k}=n} \frac{2^{-\left(j_{1}++j_{k}\right)}}{j_{1} ! \cdots j_{k} !} B_{i_{1}}^{j_{1}} \cdots B_{i_{k}}^{j_{k}} \\
& =\sum_{n=0}^{\infty}(-1)^{n} \sum_{\substack{i_{1}<<i_{k} \\
i_{1} j_{1}+\\
+i_{k} k_{k}=n}} \frac{2^{-\left(j_{1}++j_{k}\right)}}{j_{1} ! \cdots j_{k} ! i_{1}^{j_{1}} \cdots i_{k}^{j_{k}}} A_{i_{1}}^{j_{1}} \cdots A_{i_{k}}^{j_{k}} .
\end{aligned}
$$

Comparing (3.25) and (3.28) we get the last statement of the lemma.

We arrive now at the main theorem of this section considering the general case where $B, B^{-1} \in \mathscr{L}(\mathscr{H}), B>0$.

Theorem. Let $V(x)=\int \exp \{i(x, \alpha)\} d \mu(\alpha)$ and $g(x)=\int \exp \{i(x, \beta)\} d v(\beta)$, where $\mu$ and $v$ are bounded complex measures on $\mathscr{H}$ such that

$$
\begin{aligned}
& \int|\alpha|^{n} d|\mu|(\alpha) \leqq L n ! / \varepsilon^{n}, \quad 0<n, \\
& \int|\beta|^{n} d|v|(\beta) \leqq M n ! / \varepsilon^{n}, \quad 0 \leqq n,
\end{aligned}
$$

for some $L, M, \varepsilon>0$ verifying $2 L\left\|B^{-1}\right\|(3+2 \sqrt{2})<\varepsilon^{2}$. Then there is a unique point $a \in \mathscr{H}$ such that $d V(a)=B a ; B^{-1} d^{2} V(a)$ is of trace class and $\left\|B^{-1} d^{2} V(a)\right\|_{1}<1$. Let

$$
I(h)=\int_{\mathscr{H}} \exp \left\{\frac{i}{2 h}(x, B x)-\frac{i}{h} V(x)\right\} g(x) d x .
$$

Then $I(h)$ is analytic in $\operatorname{Im} h<0$. Consider the function

$$
\begin{aligned}
& I^{*}(h)=I(h) \exp \left\{\frac{i}{h} V(a)-\frac{i}{2 h}(a, B a)\right\}, \quad \text { for } \operatorname{Im} h \leqq 0, h \neq 0, \\
& I^{*}(0)=\operatorname{Det}\left(1-B^{-1} d^{2} V(a)\right)^{-1 / 2} g(a),
\end{aligned}
$$

where $\operatorname{Det}\left(\mathbb{1}-B^{-1} d^{2} V(a)\right)$ is the Fredholm determinant of the operator $\mathbb{1}$ $B^{-1} d^{2} V(a)$. Then $I^{*}(h)$ is a continuous function of $h$ in $\operatorname{Im} h \leqq 0$. One has the following asymptotic expansion and estimate

$$
\begin{aligned}
\mid I^{*}(h) & -\sum_{m=0}^{l-1} h^{m}\left(-\frac{i}{2}\right)^{m} \sum_{n=0}^{\infty} \frac{(-2)^{-n}}{n !(m+n) !} \\
& \cdot \int \cdots \int\left\{B^{-1 / 2}\left(\sum_{j=1}^{n} \alpha_{j}+\beta\right)\right\}_{(\alpha, 2)}^{2 m+2 n} \prod_{j=1}^{n} \exp \left\{i\left(a, \alpha_{j}\right)\right\} d \mu\left(\alpha_{j}\right) \exp \{i(a, \beta)\} d v(\beta) \mid \\
= & \mid I^{*}(h)-\operatorname{Det}\left(\mathbb{1}-B^{-1} d^{2} V(a)\right)^{-1 / 2} \sum_{m=0}^{l-1} h^{m}\left(-\frac{i}{2}\right)^{m} \sum_{n=0}^{2 m} \frac{2^{-n}}{n !(m+n) !}
\end{aligned}
$$




$$
\begin{aligned}
& \cdot \int \cdots \int\left\{\left(B-d^{2} V(a)\right)^{-1 / 2}\left(\sum_{j=1}^{n} \alpha_{j}+\beta\right)\right\}_{(\alpha, 3)}^{2 m+2 n} \\
& \cdot \prod_{j=1}^{n} \exp \left\{i\left(a, \alpha_{j}\right)\right\} d \mu\left(\alpha_{j}\right) \exp \{i(a, \beta)\} d v(\beta) \mid \\
& \leqq \\
& (2-\sqrt{2}) \sqrt{\pi}\left(\frac{2|h|\left\|B^{-1}\right\|}{\varepsilon^{2}(6-4 \sqrt{2})}\right)^{l}\left(1-\frac{2 L\left\|B^{-1}\right\|(3+2 \sqrt{2})}{\varepsilon^{2}}\right)^{-l-1 / 2}\left(l-\frac{1}{2}\right) !
\end{aligned}
$$

Moreover the asymptotic expansion is Borel summable and determines $I^{*}(h)$ uniquely.

Proof. The uniqueness of the stationary point follows from Lemma 3 and from the assumption $2 L\left\|B^{-1}\right\|(3+2 \sqrt{2})<\varepsilon^{2}$. We remark also that this condition implies the one of Lemma 6 . This condition and Lemma 2 imply that $B^{-1} d^{2} V(a)$ is of trace class and $\left\|B^{-1} d^{2} V(a)\right\|_{1}<1$. Now $I(h)$ can be written

$$
I(h)=\sum_{n=0}^{\infty}\left(-\frac{i}{h}\right)^{n} \frac{1}{n !} \int \cdots \int \exp \left\{-\frac{i h}{2}\left[B^{-1 / 2}\left(\sum_{j=1}^{n} \alpha_{j}+\beta\right)\right]^{2}\right\} \prod_{j=1}^{n} d \mu\left(\alpha_{j}\right) d v(\beta),
$$

using the properties of the normalized-oscillatory integral. This expression shows that $I(h)$ is analytic in $\operatorname{Im} h<0$ and continuous in $\operatorname{Im} h \leqq 0, h \neq 0$.

We can now prove that

$$
I^{*}(h)=\sum_{n=0}^{\infty} I_{n}^{*}(h)
$$

in $\operatorname{Im} h \leqq 0$, with $I_{n}^{*}(h)$ defined as before. In fact this series is absolutely and uniformly convergent in $\operatorname{Im} h \leqq 0$, and every term is analytic in $\operatorname{Im} h<0$ and $C^{\infty}$ on $\mathbb{R}$. Therefore the sum of this series is an analytic function on $\operatorname{Im} h<0$ and continuous on $\mathbb{R}$. By Lemma 4 this sum is identified with $I^{*}(h)$ in $\operatorname{Im} h<0$. For this reason $I^{*}(h)$ is just the sum of the series in $\operatorname{Im} h \leqq 0$. Now using Lemma 5 and Lemma 6 we get the first expression of the asymptotic expansion as well as the estimate of the theorem.

In order to obtain the second expression of the asymptotic expansion let us begin with another integral representation for $W(x)$. From (3.5) and (3.6) we have

$$
\begin{aligned}
& W(x)=(1 / 2)\left(x, d^{2} V(a) x\right)+\tilde{W}(x), \text { where } \\
& \tilde{W}(x)=\frac{1}{2} \int_{0}^{1} d t \int(i(x, \alpha))^{3}(1-t)^{2} \exp \{i t(x, \alpha)\} d \mu^{a}(\alpha) .
\end{aligned}
$$

Hence $(1 / 2)(x, B x)-W(x)=(1 / 2)(x, T x)-\tilde{W}(x)$, where $T=B-d^{2} V(a)$. From the Cameron-Martin formula [7], we get

$$
I^{*}(h)=\operatorname{Det}\left(\mathbb{1}-B^{-1} d^{2} V(a)\right)^{-1 / 2} \tilde{\int} \exp \left\{\frac{i}{2 h}(x, T x)-\frac{i}{h} \tilde{W}(x)\right\} g^{a}(x) d x,
$$

where the integral is normalized relative to the quadratic form $(x, T x)$. In the same way as in Lemma 4 one has in the region $\operatorname{Im} h<0$,

$$
I^{*}(h)=\operatorname{Det}\left(1-B^{-1} d^{2} V(a)\right)^{-1 / 2} \sum_{n=0}^{\infty}\left(-\frac{i}{h}\right)^{n} \frac{1}{n !} \tilde{\int} \exp \left\{\frac{i}{2 h}(x, T x)\right\} \tilde{W}(x)^{n} g^{a}(x) d x
$$


As in the proof of Lemma 5 we get

$$
\begin{aligned}
I^{*}(h)= & \operatorname{Det}\left(\mathbb{1}-B^{-1} d^{2} V(a)\right)^{-1 / 2} \sum_{n=0}^{\infty}\left(\frac{i}{h}\right)^{n} \frac{2^{-n}}{n !} \int \cdots \int \exp \left\{-\frac{i h}{2}\left(A_{n}, T^{-1} A_{n}\right)\right\} \\
& \cdot H_{3 n}\left(1-t_{1}\right)^{2} \cdots\left(1-t_{n}\right)^{2} \prod_{j=1}^{n} d \mu^{a}\left(\alpha_{j}\right) d t_{j} d v^{a}(\beta),
\end{aligned}
$$

where $A_{n}=\alpha_{1} t_{1} \cdots+\alpha_{n} t_{n}+\beta$, and

$$
H_{3 n}=H_{3 n}\left(\left(\left(\frac{i h}{2}\right)^{1 / 2} T^{-1 / 2} \alpha_{1}\right)^{3}, \ldots,\left(\left(\frac{i h}{2}\right)^{1 / 2} T^{-1 / 2} \alpha_{n}\right)^{3} \mid\left(\frac{i h}{2}\right)^{1 / 2} T^{-1 / 2} A_{n}\right) .
$$

Comparing this and (2.5) we obtain for $\operatorname{Im} h<0$

$$
\begin{aligned}
I^{*}(h)= & \operatorname{Det}\left(1-B^{-1} d^{2} V(a)\right)^{-1 / 2} \sum_{n=0}^{\infty}\left(\frac{i}{h}\right)^{n} \frac{1}{n !} \int \cdots \int \\
& \cdot \exp \left\{-\frac{i h}{2}\left[T^{-1 / 2}\left(\sum_{j=1}^{n} \alpha_{j}+\beta\right)\right]^{2}\right\} \prod_{(\alpha, 3)}^{n} d \mu^{a}(\alpha) d v^{a}(\beta) .
\end{aligned}
$$

We can now identify the coefficients of $h^{m}$, which are uniquely determined and do not depend on the domain of definition of $h$, and obtain the second expression of the asymptotic expansion of the theorem.

Finally, as we have established $I^{*}(h)$ is analytic in the half-plane $\operatorname{Im} h<0$ and satisfies there an estimate of the type

$$
\left|I^{*}(h)-\sum_{n=0}^{l-1} a_{m} h^{m}\right| \leqq A \sigma^{l} l !|h|^{l},
$$

(for $a_{m} \in \mathbb{C}, m \in \mathbb{N}, A, \sigma>0$ ) uniformly in $l \in \mathbb{N}$ and $h$. Following Nevanlinna $[14,15]$ these are precisely sufficient conditions in order to have the Borel summability of the divergent series $\Sigma a_{m} h^{m}$ and in order that $I^{*}(h)$ be uniquely determined by it. This last assertion means that there is one and only one $I^{*}(h)$ which verifies the above inequalities; this follows also from Carleman's theorem [16].

Remark. The definition of asymptotic development was introduced by H. Poincaré [17]. The theory of divergent series was first considered by E. Borel $[18,19]$. Important theorems on Borel summability were proved by G. N. Watson [20,21]. About the work of Watson, see also ref. [22]. The most powerful theorems on this subject were proved by F. Nevanlinna [14]. A good summary of Nevanlinna's results was given in ref. [15]; see also ref. [16,23]. About asymptotic developments, see also ref. [24].

Acknowledgements. I thank the referee for valid suggestions he made in order to improve the presentation of this paper.

I am grateful to Profs. S. Albeverio and Ph Blanchard for constant stimulating discussions and suggestions. Financial support by the DAAD (Deutscher Akademischer Austauschdienst) is gratefully acknowledged. 


\section{References}

1. Albeverio, S., Hфegh-Krohn, R.: Mathematical theory of Feynman Path integrals. Lecture Notes in Mathematics, Vol. 523. Berlin, Heidelberg, New York: Springer 1976

2. Albeverio, S., Hфegh-Krohn, R.: Oscillatory integrals and the method of stationary phase in infinitely many dimensions, with applications to the classical limit of quantum mechanics I Invent. Math. 40, 59-106 (1977)

3. Albeverio, S., Hфegh-Krohn, R.: Feynman Path integrals and the corresponding method of stationary phase Lecture Notes in Physics, Vol 106, pp 3-57. Berlin, Heidelberg, New York: Springer 1979

4. Albeverio, S., Blanchard, Ph., H $\phi$ egh-Krohn, R.: Feynman Path integrals and the trace formula for the Schrödinger operators. Commun. Math. Phys. 83, 49-76 (1982)

5. Rezende, $\mathrm{J}$ : Remark on the solution of the Schrödinger equation for anharmonic oscillators via the Feynman Path integral. Lett. Math. Phys. 7, 75-83 (1983)

6 Truman, A.: The polygonal Path formulation of the Feynman Path integral Lecture Notes in Physics, Vol. 106, pp. 73-102. Berlin, Heidelberg, New York: Springer 1979

7. Elworthy, D., Truman, A.: Feynman maps, Cameron-Martin formulae and an harmonic oscillators Ann. Inst Henri Poincare 41, 115-142 (1984)

8. Hörmander, L.: Fourier integral operators I Acta Math. 127, 79-183 (1971)

9. Fedoriuk, M. V.: The stationary phase method and pseudodifferential operators. Russ Math. Surv. 26, 65-115 (1971)

10. Maslov, V. P., Fedoriuk, M. V.: Semi-classical approximation in quantum mechanics Dordrecht: D. Reidel Publishing Company 1981

11. Schilder, M.: Some asymptotic formulas for Wiener integrals. Trans. Am. Math. Soc 125, 63-85 (1966)

12. Watson, G. N.: An expansion related to Stirling's formula, derived by the method of steepest descents. Q. J. Pure. Appl Math. 48, 1-18 (1920)

13. Riordan, J.: Combinatorial identities. New York: John Wiley 1968

14. Nevanlinna, F.: Zur Theorie der asymptotischen Potenzreihen Ann. Acad. Sci. Fenn. (A) 12, no 3 (1976)

15. Bieberbach, L.: Jahrb, Forfschr Math. 46, 1463-1465 (1916-1918)

16. Simon, B.: Large orders and summability of eigenvalue perturbation theory: A mathematical overview. Int. J. Quant. Chem. 21, 3-25 (1982)

17. Poincaré, H.: Sur les intégrales irrégulières des equations linéaires. Acta Math. 8, 295-344 (1886)

18. Borel, E.: Oeuvres, pp. 399-568. Paris: C.N.R.S 1972

19. Borel, E.: Mémoire sur les séries divergentes Ann. Ec. Norm. Sup. $3^{e}$.série, $t$. 16, 9-131 (1899)

20. Watson, G. N.: The transformation of an asymptotic series into a convergent series of inverse factorials. Rend. Circ. Mat. Palermo 34, 41-88 (1912)

21. Watson, G. N.: A theory of asymptotic series. Trans. Royal Soc. London A 211, 279-313 (1912)

22. Hardy, G. H.: Divergent series London: Oxford University Press 1963

23. Sokal, A.: An improvement of Watson's theorem on Borel summability. J Math Phys. 21, 261-263 (1980)

24. Dieudonné, J.: Calcul infinitésimal. Paris: Hermann 1968

Communicated by $\mathrm{H}$. Araki

Received June 19, 1984; in revised form December 25, 1984 